\title{
O.S.P.
}

L'orientation scolaire et professionnelle

$34 / 3 \mid 2005$

Émotions et transitions

\section{Marcel Bromberg \& Alain Trognon (2004). Psychologie sociale et communication}

Paris : Dunod

Isabelle Olry-Louis

\section{OpenEdition}

Journals

Édition électronique

URL : https://journals.openedition.org/osp/793

DOI : $10.4000 /$ osp.793

ISSN : 2104-3795

Éditeur

Institut national d'étude du travail et d'orientation professionnelle (INETOP)

Édition imprimée

Date de publication : 1 septembre 2005

ISSN : 0249-6739

\section{Référence électronique}

Isabelle Olry-Louis, « Marcel Bromberg \& Alain Trognon (2004). Psychologie sociale et

communication », L'orientation scolaire et professionnelle [En ligne], 34/3 | 2005, mis en ligne le 28

septembre 2009, consulté le 18 janvier 2022. URL : http://journals.openedition.org/osp/793 ; DOI

https://doi.org/10.4000/osp.793

Ce document a été généré automatiquement le 18 janvier 2022.

(c) Tous droits réservés 


\section{Marcel Bromberg \& Alain Trognon (2004). Psychologie sociale et communication}

Paris : Dunod

Isabelle Olry-Louis

\section{RÉFÉRENCE}

Marcel Bromberg \& Alain Trognon (2004). Psychologie sociale et communication. Paris :

Dunod.

1 Cet ouvrage est d'abord un hommage à Rodolphe Ghiglione. Il fait suite au colloque qui s'est tenu en février 2001 à l'Université Paris VIII en psychologie sociale de la communication, domaine de recherche initié et durablement marqué par les travaux de R. Ghiglione. L'originalité de son approche a consisté, dès les années 70 , à considérer le langage, non pas comme un phénomène transparent, un simple miroir, mais plutôt comme une source de questionnement. Quelles sont les spécificités des situations de communication dans lesquelles il se déploie? Quelles relations unissent les activités cognitives du sujet communiquant et son langage? Par quels processus les échanges interlocutoires sont ils réglés? Et avec quels effets? Voilà quelques questions auxquelles se sont consacrés R. Ghiglione, mais aussi tous les chercheurs issus de Paris VIII ou d'ailleurs qu'il a su, au fil du temps, rassembler autour de sa personne pour tenter de définir ce que communiquer veut dire. Le concept nodal de contrat de communication a constitué un élément de réponse autour duquel s'est cristallisée la réflexion visant à saisir dans sa totalité les paramètres de l'interaction communicative. Plusieurs recherches ici relatées s'inscrivent dans la filiation directe de cette perspective.

2 Toutefois, cet ouvrage ne porte pas directement sur les travaux de R. Ghiglione. Il en constitue plutôt un prolongement, au sens où il tente "d'organiser une communauté 
d'intérêt dans le champ très large de la psychologie de la communication » comme le signalent Marcel Bromberg et Alain Trognon dans leur préface. Il s'agit en effet pour eux d'initier, à travers un ouvrage collectif, une dynamique de collaboration entre chercheurs appartenant à des champs et à des disciplines variés concernant la problématique de l'usage du langage dans des situations d'interaction sociale. Ce pari est tenu, sans conteste. L'ouvrage en lui-même atteste, par la richesse, la diversité et l'articulation de son contenu, la présence d'un champ de recherche actif et mature tant au plan théorique, qu'aux plans empirique et expérimental. Sans prétendre constituer un manuel, il pourra remplir cette fonction pour l'étudiant avancé qui s'intéresse aux questions de communication et trouvera là un ensemble de contributions, une bibliographie assez complète et des index d'auteurs et de notions fort utiles. Les chercheurs, qu'ils soient psychologues, linguistes, sociologues ou pragmaticiens, disposent avec cet ouvrage d'un panorama de recherches sur la communication qui, même si elles s'inscrivent principalement dans le champ de la psychologie sociale nous y reviendrons -, présentent un intérêt évident pour l'ensemble des problématiques de communication. Enfin les praticiens, notamment ceux, enseignants, conseillers, psychothérapeutes, psychologues, travailleurs sociaux qui exercent leur activité par le langage, et tous ceux qui conçoivent des campagnes de communication sont bien évidemment les cibles de choix auxquels s'adressent cet ouvrage.

3 Venons-en à la structure. Ce n'est pas celle d'un simple recueil d'actes de colloque, mais bien celle d'un livre. Constitué - outre la préface, la bibliographie et les index déjà signalés-d'une introduction consistante et de 19 chapitres, d'une douzaine de pages en moyenne chacun, qui sont organisés en 4 parties intitulées « la communication comme symptôme ", «les contrats de communication ", « les interactions communicatives » et "les schémas de communication", l'ouvrage est très bien présenté tant au plan matériel qu'au plan du contenu. Sans prétendre rendre compte de l'ensemble des contributions, nous nous attarderons sur quelques-unes d'entre elles qui nous ont paru particulièrement significatives.

4 Dans leur texte introductif, Marcel Bromberg et Alain Trognon rappellent les conditions historiques de la constitution de la psychologie sociale de la communication, puis esquissent, à partir d'une caractérisation des recherches figurant dans l'ouvrage, un programme de développement pour cette branche. Partant de la définition première des phénomènes de communication proposée par Moscovici en 1984 comme étant «les échanges de messages linguistiques et non linguistiques (images, gestes, ...) entre individus et groupes ... utilisés pour transmettre une certaine information et influencer autrui», ils soulignent aujourd'hui l'importance, pour toute communication verbale, des mécanismes inférentiels au côté des mécanismes de décodage plus classiquement établis, et précisent que le jeu social d'influence a presque toujours une visée persuasive. De même que l'on est passé d'une conception du sujet-réceptacle (proie désignée et passive du piège argumentatif) au modèle des vases communicants, l'on va peu à peu considérer qu'il n'y a pas d'acte de communication qui ne renvoie simultanément à un transfert d'information et à l'établissement d'une relation, tout sujet étant susceptible de réagir non seulement au message mais aussi à la relation. En somme, le sujet social va passer du statut d'émetteur-récepteur, à celui de locuteurinterlocuteur doté de mécanismes cognitifs qui anticipe et produit (au moins en pensée) des stratégies argumentatives et contre-argumentatives. 
5 Les recherches présentées dans l'ouvrage peuvent être distinguées selon qu'elles portent sur des propriétés relativement générales de la communication ou qu'elles s'attachent à spécifier des situations ou des contrats de communication. Nous décrirons brièvement chacun de ces ensembles de travaux avant de les illustrer à travers un exemple détaillé.

6 Avec le premier type de recherches, que M. Bromberg et A. Trognon qualifient de "microscopiques", trois idées sont mises en avant. Tout d'abord, les propriétés syntaxico-sémantiques des messages "réfléchissent» les cognitions sociales des émetteurs qui les produisent, ce que développent dans leurs chapitres Philippe Castel et Marie-Françoise Lacassagne à propos des discours de discrimination raciale, ou Edith Sales-Wuillemin et Daniel Gilibert à propos des biais d'attribution causale. En sens inverse, la matière même de la communication contribue au façonnage des cognitions des participants. Quatre exemples peuvent illustrer cette thèse. Selon Pascal Marchand, le contexte et la nature de l'interaction peuvent rendre fluctuantes les représentations socio-politiques des individus. Dans une perspective analogue, Patrice Georget fait la démonstration que les signaux paraverbaux (intonation, expression faciale, direction du regard) accompagnant le discours d'hommes politiques modulent la crédibilité accordée à leur message, pourtant identique. De leur côté, Annette Burguet et Denis Hilton défendent l'idée selon laquelle l'attribution causale doit certes être interprétée en fonction de facteurs sémantiques comme la logique des verbes mais doit aussi s'ouvrir à une analyse plus pragmatique de ces processus, une attention toute particulière devant être accordée aux effets de contexte. Dans un dernier exemple, Thierry Meyer et Éric Yonnet démontrent en manipulant des variables linguistiques (le cadrage des arguments, leur modélisation dans le message et l'implication de leurs cibles) que l'introduction d'une modalisation de doute dans les arguments produits par la source change quantitativement et qualitativement les prédictions issues des modèles de persuasion. La troisième idée soulignée dans cette série de recherches c'est que l'activité conversationnelle contribue d'autant mieux au façonnage des cognitions qu'elle fait suite à un comportement préparatoire consistant avec les arguments véhiculés par message persuasif.

7 Pour l'illustrer, arrêtons-nous un moment sur le chapitre de Robert-Vincent Joule, Jacques Py et Françoise Bernard intitulé «Qui dit quoi, à qui, en lui faisant faire quoi ? Vers une communication engageante ». À travers la notion de communication engageante, les auteurs tentent de jeter les bases d'un dialogue entre la psychologie sociale et les sciences de l'information et de la communication. Revenons d'abord sur le concept d'engagement. Défini initialement par Kiesler comme « le lien qui unit l'individu à ses actes comportementaux ", l'accent est mis ici sur les conditions dans lesquelles, pour une situation donnée, la réalisation d'un acte ne peut être imputée qu'à celui qui l'a réalisé. Les résultats expérimentaux du paradigme du pied dans la porte, bien connus, conduisent à penser qu'un message persuasif a plus de chances de déboucher sur les effets escomptés s'il a été précédé par un acte préparatoire engageant et consistant avec lui. L'enjeu devient alors de chercher à mieux articuler théorie de la persuasion et théorie de l'engagement, ce que les auteurs parviennent à faire avec les deux études à visée d'application dont ils rendent compte. La première concerne une opération de prévention routière, la seconde est une campagne de communication mise en œuvre par une municipalité dans le but d'inciter les habitants à faire des économies d'énergie. À chaque fois, il s'agit de conférer au récepteur de l'information un statut d'acteur en 
l'amenant à réaliser des actes peu coûteux qui l'engagent - accrocher un foulard à l'antenne de son véhicule en témoignage de la volonté de respecter le code de la route dans le premier cas, répondre au téléphone à une enquête sur la consommation d'énergie, aller retirer une brochure d'information et signer un engagement personnalisé dans le second cas - et le préparent ainsi à mieux accepter les messages éducatifs, préventifs qui lui seront adressés ultérieurement.

8 L'autre ensemble de recherches présentées dans cet ouvrage, qualifiées de «macroscopiques» par M. Bromberg et A. Trognon, décrit des jeux d'interaction en situation. Les situations de communication sont définies par Patrick Charaudeau comme des cadres de contraintes psychosociales qui imposent des instructions quant à la façon de mettre en scène le discours. Elles peuvent être caractérisées par leurs finalités, par l'identité de leurs participants, par leurs propos et par les circonstances. Les échanges recueillis par Michèle Grosjean dans les centres opérationnels du R.E.R. s'inscrivent ainsi dans une situation de communication tout à fait spécifique. Certaines situations se traduisent aussi en formats typiques plus ou moins réguliers, comme ceux auxquels donnent lieux ces communications aux confins de la normalité que sont les conversations schizophréniques abordées au plan général par Alain Blanchet - avec les notions d'incompétence pragmatique, d'incohérence textuelle, d'impertinence conversationnelle, d'apraxie cognitive - et sur le plan plus particulier des discours sur le monde par Michel Musiol. Les situations de communication s'expriment aussi quelquefois sous forme de contrats de communication. Le contrat est, selon P. Charaudeau, «ce qui parle avant que quiconque ait parlé, ce qui est compris avant même qu'on l'ait lu ", grâce au système de reconnaissance réciproque des interlocuteurs ou du producteur et du récepteur du message écrit. Le contrat c'est aussi, selon Odile Camus, ce qui « rend compte du déterminisme qu'exercent les caractéristiques des situations de communication (buts et enjeux de la rencontre, statuts des partenaires), sur la structure et le contenu des échanges ». Plusieurs chapitres sont consacrés à décrire des contrats de communication. Ainsi, Giuseppe Minnini, partant d'une analyse conversationnelle des propos échangés dans le cadre de " Newsgroups » ou de "Chat ", désigne par «contrat de communication virtuelle» le fait que «la communication modelée par Internet oblige les sujets à négocier la révélation d'aspects multiples de soi et la prise en charge de différentes positions discursives, de sorte que l'individu est porté à marquer les traces aussi bien de son identification que de sa différenciation ". Laurence Masse compare les contrats de communication relatifs à deux types de thérapies de face à face: une thérapie cognitive et une thérapie d'inspiration psychanalytique. Elle montre ainsi que la clef du changement thérapeutique se situe dans une transmission explicite d'attitudes en thérapie cognitive et dans des jeux d'implicite en thérapie d'inspiration psychanalytique. Alain Trognon et Martine Batt analysent pour leur part un épisode d'une consultation médicale prédictive de maladie génétique, au cours duquel une conviction propre à une patiente est combattue par un médecin.

9 Arrêtons-nous un moment sur le contrat de communication propre à l'entretien de recrutement, sur la base du chapitre que 0 . Camus lui consacre. Cet entretien constitue une caricature des interactions quotidiennes du fait même de la visibilité des enjeux identitaires qu'il véhicule. Il s'agit pour le candidat de « faire bonne figure » dans « une situation embarrassante ", ce qui passe par la mise en scène d'une image positive de soi en tant que sujet employable. Au plan interactionnel, les partenaires de l'échange occupent une position assymétrique, la supériorité positionnelle du recruteur étant 
attestée, au plan communicationnel, par un rôle de questionnant, le candidat étant quant à lui invité à prendre en charge un important capital verbal lors de séquences thématiques convenues (motivation pour le poste, auto-description personnologique en terme de défauts et de qualités). Le contrat de communication est ambigu au sens où le candidat peut l'interpréter de deux manières : considérer que les aspects informatifs qu'il fournira constituent les éléments décisifs sur lesquels le recruteur s'appuiera pour prendre sa décision, ou bien considérer qu'il s'agit surtout d'une épreuve de communication destinée à appréhender certaines «qualités sociales ", et qu'il importe plus de soigner son comportement d'interactant que le contenu de son discours. L'auteur relate plusieurs études qu'elle a menées dans ce champ. Dans l'une d'elles, elle manipule la position communicationnelle du recruteur à partir de variations spontanées qu'elle a observées chez les praticiens et retient deux modalités : la position consensuelle, marquée par l'écoute, par la non-directivité et par un rôle communicationnel de validant versus la position provocatrice marquée par l'émission de signaux dubitatifs, de désaccords, d'interruptions et par un rôle communicationnel d'évaluant-invalidant. Les autodescriptions qu'elle recueille chez les candidats apparaissent moins stéréotypées et plus discriminantes lorsque le recruteur se montre provocateur. C'est aussi dans ce cas qu'elle observe une plus grande proximité entre les partenaires, la position consensuelle ayant pour effet de différencier d'emblée les rôles des partenaires, le candidat fournissant de l'information et le recruteur prenant en charge la gestion de la rencontre. Une autre étude consiste à confronter les critères de l'évaluation définis par le recruteur lui-même à l'analyse systématique des entretiens. Elle montre que les candidats qui seront sélectionnés à l'issue de l'entretien sont en fait ceux qui objectivement répondent le moins aux critères définis. Leur discours est peu structuré et non argumentatif, ils parlent surtout d'eux-mêmes, la vie extraprofessionnelle occupant une place relativement importante par rapport au parcours professionnel. Mais l'alternance de la parole est rapide, avec une bonne symétrie des rôles communicationnels. Tout se passe comme si «le bon candidat» était celui qui savait établir une connivence avec le recruteur, en conférant à ce dernier une plus forte présence communicationnelle que ne le permettrait un contrat de communication strictement informatif.

10 Avec ces exemples, le lecteur aura compris que les jeux d'interaction dont la psychologie sociale est en train d'élaborer les instruments d'analyse sont des pratiques sociales réelles. Qu'il s'agisse d'entretiens de recrutement, d'entretiens thérapeutiques ou de campagnes de communication, ils constituent autant de situations d'interaction sociale dans lesquelles la seule réalité qui nous est donnée à voir à propos d'événements ou de personnes n'est pas tant la réalité vécue qu'une version linguistique particulière de cette réalité. Le langage ordonne nos perceptions du monde, aussi toute réflexion sur la communication doit-elle montrer comment le langage peut être utilisé pour construire l'interaction sociale dans différents mondes sociaux. Ce n'est pas le moindre mérite de cet ouvrage d'ouvrir ici la réflexion à de véritables champs de pratiques sociales.

11 En somme, cet ouvrage collectif pourrait bien devenir un livre de référence dans le domaine parce qu'il marque les bases théoriques et empiriques d'un champ en pleine expansion d'une part et parce ce qu'il présente de réelles qualités de présentation matérielle, de lisibilité et de cohérence d'autre part. De toute évidence le propos est unifié à travers les contributions. Rend-il pour autant compte de l'avancée d'une communauté d'intérêt dans le champ de la psychologie de la communication? Si l'on 
apprécie que plusieurs approches soient conjuguées pour rendre compte d'un même ensemble de phénomènes - on notera en particulier l'apport important constitué par les approches psychopathologiques du langage -, l'on peut aussi regretter l'absence ou la faible représentation de disciplines qui s'efforcent aujourd'hui de repenser leurs concepts et leurs méthodes à partir de problématiques propres à la communication. C'est le cas de la psychologie du développement, jamais évoquée dans le volume, en dépit de son intense activité dans le champ et du rayonnement dont elle fait preuve, notamment par le truchement des concepts qu'elle contribue à élaborer, comme celui de théories de l'esprit, d'ailleurs central dans plusieurs des contributions de l'ouvrage. La psychologie sociale du développement n'apparait pas davantage ici, alors que les travaux qu'elle a initiés et développés sur la question du conflit socio-cognitif et plus généralement sur celle des interactions à visée d'apprentissage sont très pertinents pour interroger la relation entre interactions sociales et cognition. Le point de vue de la psychologie du travail enfin est à notre sens insuffisamment développé dans l'ouvrage, un seul chapitre étant consacré aux collectifs de travail, domaine actuellement très investi par tous ceux qui s'intéressent aux rapports qu'entretiennent le langage et l'activité dans le travail. Mais l'objectif de l'ouvrage, rappelons-le, était double : rendre hommage à Rodolphe Ghiglione, avec ses proches collaborateurs, et inscrire la psychologie sociale dans un champ de recherches ouvert autour de la communication. Le lecteur constatera que, si le premier objectif est totalement satisfait, le second est mieux rempli dans le cadre de la psychologie sociale, que dans celui de son articulation avec la psychologie de la communication, ce qui, après tout, correspond précisément au titre de l'ouvrage. 